\title{
Arctigenin Attenuates Ischemia/ Reperfusion Induced Ventricular Arrhythmias by Decreasing Oxidative Stress in Rats
}

\author{
Jing Yang ${ }^{\mathrm{a}}$ Hong-shan Yin ${ }^{\mathrm{b}}$ Ya-jing Caoc ${ }^{c}$ Zhi-an Jiang ${ }^{\mathrm{b}}$ Yong-jun Li $^{\mathrm{d}}$ \\ Mu-chun Songe Yong-fei Wang ${ }^{e}$ Zhi-hua Wang ${ }^{f}$ Rong Yang ${ }^{d}$ Yun-fa Jiang ${ }^{d}$ \\ Jin-peng Sung Bo-yi Liu ${ }^{\text {h }}$ Chuan Wang ${ }^{f}$
}

\begin{abstract}
aDepartment of Physiology, Hebei Medical University, Shijiazhuang, 'Department of Cardiology, the Third Hospital of Hebei Medical University, Shijiazhuang, 'Department of Chronic Noncommunicable Disease Control and Prevention, Hebei Provincial Center for Disease Control and Prevention. Shijiazhuang, dDepartment of Cardiology, the Second Hospital of Hebei Medical University, Shijiazhuang, ${ }^{e}$ College of Basic Medicine, Hebei Medical University, Shijiazhuang, ${ }^{f}$ Department of Pharmacology, Hebei Medical University, Shijiazhuang, ${ }^{9}$ Key Laboratory Experimental Teratology of the Ministry of Education and Department of Biochemistry and Molecular Biology, Shandong University School of Medicine, Jinan, h Department of Neurobiology and Acupuncture Research, the Third Clinical Medical College, Zhejiang Chinese Medical University, Hangzhou, China
\end{abstract}

\section{Key Words}

Arctigenin • Arrhythmias • Ischemia reperfusion • Oxidative stress • Nrf2 signaling

\begin{abstract}
Background/Aims: Arctigenin (ATG) has been shown to possess anti-inflammatory, immunemodulatory, anti-viral, anti-microbial, anti-carcinogenic, vasodilatory and antiplatelet aggregation properties. However, the protective role of ATG in prevention of arrhythmias induced by myocardial ischemia/reperfusion is unknown. The aim of this study was to investigate the anti-arrhythmia effect of ATG in an ischemia/reperfusion injured rat heart model and explore the related mechanisms. Methods: Rats were randomly exposed to sham operation, myocardial ischemia/ reperfusion (MI/R) alone, ATG + MI/R, pretreated with ATG in low (12.5 mg/kg/day), medium (50 mg/kg/day) and high dose (200 mg/kg/day), respectively. Ventricular arrhythmias were assessed. The activity of superoxide dismutase (SOD), glutathione peroxidase (GSH-Px) and the level of malondialdehyde (MDA) in myocardial tissue were determined by chemical analysis. Results: Compared to MI/R, rats pretreated with ATG in doses of $50 \mathrm{mg} / \mathrm{kg} /$ day and $200 \mathrm{mg} / \mathrm{kg} /$ day showed significantly reduced incidence and duration of ventricular fibrillation, ventricular tachycardia and ventricular ectopic beat (VEB), and decreased the arrhythmia score during the 30-min ischemia. Incidence and duration of \footnotetext{
Department of Cardiology, the Third Hospital of Hebei Medical University, 139 Ziqiang Road, Shijiazhuang; and Dr. Chuan Wang Department of Pharmacology, Hebei Medical University, 361 Zhongshan East Road, Shijiashuang (China) Tel. (86)18533112881, E-Mail 18533112881@163.com; wangchuan@hebmu.edu.cn
}

Dr. Hong-shan Yin
\end{abstract}




\section{Cellular Physiology Cell Physiol Biochem 2018;49:728-742 \\ \begin{tabular}{l|l|l} 
DOI: 10.1159/000493038 & $\begin{array}{l}\text { O 2018 The Author(s). Published by S. Karger AG, Basel } \\
\text { www.karger.com/cpb }\end{array}$
\end{tabular}}

Yang et al.: Arctigenin Attenuates Arrhythmias by decreasing Oxidative Stress

ventricular tachycardia, infarction size and arrhythmia scores in these groups were significantly decreased during the 120 -min reperfusion. No ventricular fibrillation occurred during the period of reperfusion. Rats pretreated with ATG in doses of $50 \mathrm{mg} / \mathrm{kg} / \mathrm{day}$ and $200 \mathrm{mg} / \mathrm{kg} /$ day markedly enhanced the activities of antioxidant enzymes SOD and GSH-Px, reduced the level of MDA. No differences were observed between the group pretreated with a low dose of ATG and the sham group. Administration of ATG significantly increased the expression of antioxidant stress protein Nrf2, Trx1 and Nox1. Conclusion: Our data suggested that ATG plays anti-arrhythmia role in ischemia/reperfusion injury, which is probably associated with attenuating oxidative stress by Nrf2 signaling pathway.

\section{Introduction}

Cardiovascular diseases are a leading cause of mortality and morbidity worldwide, in which the main clinical model of ischemia/ reperfusion injury is acute myocardial infarction [1]. Related to ischemia/reperfusion injury, redox imbalance triggers the activity of several signaling pathways mediated by reactive oxygen species (ROS) and reactive nitrogen species [2]. Oxidative stress has been suggested as a pathogenic factor and therapeutic target in early stages of essential myocardial ischemia/reperfusion injury [3]. Numerous studies have shown the contribution of reactive oxygen and nitrogen species in the pathogenesis of myocardial ischemia/reperfusion injury. As ischemia/reperfusion injury remains an under studied therapeutic target [4], there is growing interest in the evaluation of antioxidant phytochemicals capable of preventing or protecting stress of oxidative agents associated with myocardial ischemia/reperfusion injury. Arctigenin (ATG), a lignan extract from Fructus Arctii, is the dried fruit of Arctium Lappa L. It is known to possess anti-inflammatory, immunemodulatory, antiviral, antimicrobial, anticarcinogenic, vasodilatory and antiplatelet aggregation properties [5-8]. The nuclear factor erythroid 2-related factor 2 (Nrf2) is induced in response to oxidative stress, which might be caused by increased levels of endogenously generated ROSs [9]. Nrf2 induces transcription of cytoprotective enzymes, such as anti-oxidative enzyme thioredoxin 1 (Trx1), exerting a cytoprotective role $[10,11]$. The regulation of Nrf2 and Trx1 by NOX1 was confirmed by both inhibitions of endogenous NOX1 and overexpression of recombinant NOX1 protein. Previous studies reported that ATG can inhibit intracellular ROS production by increasing hemeoxygenase-1 gene expression in rat primary astrocytes [12], and inducing endothelial nitric oxide synthase and attenuating subarachnoid hemorrhage-induced vasospasm through PI3K/Akt pathway in a rat model [13]. Arctigenin even has been shown to have a neuroprotective effect by mechanisms of anti-neuroinflammation and anti-oxidative stress [14]. It is unknown if ATG has a biological effect on myocardial ischemia/reperfusion-induced ventricular arrhythmias and if it exerts antioxidative effects. Here we hypothesize ATG plays an anti-arrhythmiatic role in myocardial ischemia/reperfusion injury, and is likely associated with attenuating oxidative stress. This study investigates the effect and underlying mechanisms of ATG as an antioxidative stress therapy upon a myocardial ischemia/reperfusion rat model. Our data suggests that ATG can attenuate myocardial ischemia/ reperfusion induced ventricular arrhythmias by decreasing oxidative stress through the Nrf2 signaling pathway.

\section{Materials and Methods}

\section{Reagents}

Arctigenin and GAPDH antibodies were purchased from Sigma Chemical Co. (St. Louis, MO, USA). GSHPX and SOD activity assay kit and MDA and GSH content assay kit were purchased from Jiancheng Bioengineering Institute (Nanjing, China). Anti-Nrf2 (H-300), Trx1 (FL-105), Nox1 antibodies were purchased from Santa Cruz Biotechnology (Santa Cruz, CA, USA). 


\section{Cellular Physiology Cell Physiol Biochem 2018;49:728-742 \begin{tabular}{ll|l} 
DOI: 10.1159/000493038 & $\begin{array}{l}\text { O 2018 The Author(s). Published by S. Karger AG, Basel } \\
\text { www.karger.com/cpb }\end{array}$ \\
\hline
\end{tabular}}

Yang et al.: Arctigenin Attenuates Arrhythmias by decreasing Oxidative Stress

Animals

Adult male Sprague Dawley rats (250 -300 g) were purchased from the Center of Experimental Animal in Hebei Medical University, China. All animals used in this study were cared for in accordance with the Guide for the Care and Use of Laboratory Animals published by the United States National Institute of Healt (NIH publication no. 8523, revised 1996), and all procedures were approved by the Committee of Experimental Animals of Hebei Medical University.

The myocardial ischemia reperfusion model and experimental protocol

Male Sprague Dawley rats were anesthetized intraperitoneally with sodium pentobarbital (Sigma, St. Louis, USA, $40 \mathrm{mg} / \mathrm{kg}$ ). An intratracheal cannula was inserted and the animals were placed in intermittent positive pressure ventilation with room air. Myocardial ischemia was produced by exteriorizing the heart with a left thoracic incision followed by a slipknot (5-0 silk) around the left anterior descending coronary artery (LAD). After $30 \mathrm{~min}$ of ischemia, the slipknot was released and the animal received $120 \mathrm{~min}$ of reperfusion. A total of 50 male Sprague Dawley rats (250-300 g) were randomly assigned into 5 groups with 10 rats in each. They received the following treatments: (1) sham group (Sham): silk was drilled underneath the LAD but the LAD was not ligated; (2) myocardial ischemia/reperfusion group (MI/R): LAD was ligated for $30 \mathrm{~min}$ and then allowed 120 min reperfusion; (3) Low dose ATG + MI/R group (L-ATG + MI/R): ATG was introduced by gastric administration in a dose of $12.5 \mathrm{mg} / \mathrm{kg}$ before ischemia/ reperfusion surgery respectively for 7 days, once per day; (4) Medium dose ATG + MI/R group (M-ATG + MI/R): ATG was introduced by gastric administration in a dose of $50 \mathrm{mg} / \mathrm{kg}$ before ischemia/reperfusion surgery respectively for 7days, once per day; (5) High dose ATG + MI/R group (H- ATG + MI/R): ATG was introduced by gastric administration in a dose of $200 \mathrm{mg} / \mathrm{kg}$ before ischemia/reperfusion surgery respectively for 7 days, once per day. The sham operation group and ischemia/reperfusion group were administered $0.9 \%$ sodium chloride solution by gastric administration at the same time points.All rats were placed on an electric heating pad to maintain their body temperature at $37^{\circ} \mathrm{C}$. Heparin $(200 \mathrm{IU} / \mathrm{kg}$, i.v.) was administered before ischemia. Lead II of the electrocardiogram was monitored with subcutaneous stainless steel electrodes. A computer based EP system was used for recording MBP, heart rate and electrocardiogram. A successful myocardial ischemia model was confirmed by ST segment elevation in leads II and regional cyanosis of the myocardial surface.

\section{Assay of myocardial infarct area}

After reperfusion, myocardial infarct size was determined by means of a doublestaining technique and a digital imaging system (infarct area/ area at risk $\times 100 \%$ ] [15]. After reperfusion, coronary blood flow was again blocked and Evans blue $(2 \%, 4 \mathrm{ml})$ was injected by the rapid distribution of the right ventricle into the body. The heart was quickly isolated and cryopreserved in a $-20^{\circ} \mathrm{C}$ refrigerator. The heart was cut into 1 mm slices, placed in 1\% 2, 3,5triphenyltetrazolium chloride (TTC) solution, incubated for $15 \mathrm{~min}$, and then placed in $4 \%$ formaldehyde solution overnight. Digital images of the Evans blue stained area (blue staining, non ischemic area), TTC stained area (red staining, ischemic area) and non TTC stained area (white, infarct area) was analyzed computationally. The outcome variable, myocardial infarct area (infarct area/area at risk\%, INF/AAR\%) was calculated.

\section{Assessment of ventricular arrhythmias}

Ischemia induced ventricular arrhythmias were determined in accordance with the Lambeth Conventions $[16,17]$. Ventricular ectopic beat (VEB) was defined as identifiable premature QRS complexes (premature with respect to the P wave). Ventricular tachycardia (VT) was defined as the occurrence of four or more consecutive VEBs at a rate faster than the resting sinus rate. Ventricular fibrillation (VF) was defined as unidentifiable and low voltage QRS complexes. Other patterns of VEBs such as bigeminy, couplet (two consecutive VEBs) and salvos (three consecutive VEBs) were included in VEBs and not analyzed separately. VF may be sustained or may convert spontaneously to a normal sinus rhythm.VF resulting in death was considered as irreversible (Fig. 1).The severity of arrhythmias was quantified by the following scoring system $[16,17]$ :

- 0: 0-50 VEBs with no other arrhythmias during the 30min ischemia period

- 1: only 50-500 VEBs

- 2: more than 500 VEBs, or one episode of spontaneously reversible VT or VF

- 3: 2-30 episodes of spontaneously reversible VT and/or VF

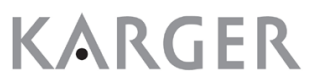




\section{Cellular Physiology Cell Physiol Biochem 2018;49:728-742 \begin{tabular}{ll|l} 
DOI: 10.1159/000493038 & $\begin{array}{l}\text { O 2018 The Author(s). Published by S. Karger AG, Basel } \\
\text { www.karger.com/cpb }\end{array}$
\end{tabular}}

Detection of SOD, GSH-Px activity and MDA content

After reperfusion, myocardial tissue was homogenized in ice cold phosphate buffer to make a $10 \%$ homogenate. The homogenate was centrifuged at $3000 \mathrm{rpm}$ for $15 \mathrm{~min}$. Superoxide dismutase (SOD), glutathione peroxidase (GSH-Px) and malondialdehyde (MDA) in the supernatant were measured by using commercially available kits. All assays were conducted according to the manufacturer's instructions.

Quantitative real-time PCR for thioredoxin 1 (Trx1) and NOX1

Quantitative real-time PCR analyses of Trx1 and NOX1 expression were performed using RNAs isolated from experimental and control samples. For quantitative real-time PCR, total RNAs were purified from hearts using Trizol reagent according to the manufacturer's instructions. RNA was transcribed to cDNA with Prime Script RT reagent Kit. qRT-PCR was performed using the CFX96TM real-time system, and the relative gene expression was normalized to internal control gapdh. The analysis of the melting curve of each amplified PCR product and the visualization of the PCR amplicons on 1.5\% agarose gels allowed to control the specificity of the amplification. Primer sequences for SYBR Green probes of target genes are as follows $(+$, forward; - , reverse):

NOX1+:CTCTCTCCTGGAATGGCATC,

NOX1-:TGGAAAACATCCTCACTGGC;

Trx1+:CAGATCGAGAGCAAGACTGCTTTTC,

Trx1-:CTTATTGGCTCCAGAAAATTCACCC;

GAPDH+:GGTGGACCTCATGGCCTACA

GAPDH-:CTCTCTTGCTCTCAGTATCCTTGCT.

Western blotting analysis of Nrf2, Trx1 and Nox1 expression

Following reperfusion, myocardial tissues were harvested and washed with ice-cold normal saline and homogenized on ice in phosphate buffered saline. After centrifugation at $3000 \mathrm{rpm}$ for $5 \mathrm{~min}$, the supernatant was discarded. The homogenate was incubated in lysis buffer including protease inhibitor, PMSF, etc. for 20 min with vortex-mixing at $4^{\circ} \mathrm{C}$, filtered and centrifuged at $12,000 \mathrm{~g}$ for $20 \mathrm{~min}$. The resulting supernatants were the proteins, of which the concentrations were determined by Bradford's method with bovine serum albumin serving as a calibrator. The immunoblots were probed with anti Nrf2 (H-300), Trx1 (FL-105), Nox1 (both Santa Cruz Biotechnology, Santa Cruz, CA, USA) and GAPDH (Abcam). Samples were incubated with these primary antibodies overnight at $4{ }^{\circ} \mathrm{C}$, and subsequently with their corresponding secondary antibodies at room temperature for $1 \mathrm{~h}$. The blots were visualized with ECL-Plus reagent.

\section{Statistical analysis}

All values were expressed as Mean \pm SE for continuous variables or the percentage of incidence of ventricular arrhythmia. A one way ANOVA or Welch was used for comparisons among groups, and the Student NeumanKeuls or Dunnett T3 was used for posthoc multiple comparisons. Paired samples t-tests were used for comparisons of heart rate and MBP within each group. Mann Whitney U test was used for comparisons of arrhythmia scores between two groups. When more than two groups were compared, Kruskale Wallis $\mathrm{H}$ test was used. Incidences of ventricular arrhythmias (i.e. VT and VF) were used for comparisons by Fisher's exact test. All statistical tests were two sided, and $\mathrm{P}<0.05$ was considered to be statistically significant. Statistical analysis was performed using SPSS 13.0 software (SPSS, Chicago, IL, USA).

Table 1. The number of episodes of ventricular ectopic beat (VEB) within 30-min ischemia. Data were expressed as mean $\pm S E$ ( $n=10$ in each group). VEB, ventricular ectopic beat. ATG, arctigenin. ${ }^{*} P<0.05$ vs. the sham group. ${ }^{*} \mathrm{P}<0.01$ vs. the sham group, ${ }^{\# \#} \mathrm{P}<0.01$ vs. MI/R group

\begin{tabular}{lccc}
\hline & Bigminy $(\mathrm{n}=10)$ & Couplet $(\mathrm{n}=10)$ & Salvos $(\mathrm{n}=10)$ \\
\hline Sham & $1 \pm 0.26$ & $0 \pm 0$ & $0 \pm 0$ \\
MI/R & $317.3 \pm 21.87^{* *}$ & $33.4 \pm 2.65^{* *}$ & $22.7 \pm 2.58^{* *}$ \\
L-ATG+ MI/R & $169.1 \pm 15.06^{* * \# \#}$ & $15.5 \pm 1.83^{* * \# \#}$ & $14.6 \pm 2.12^{* *}$ \\
M-ATG+ MI/R & $82.3 \pm 14.61^{* * \# \#}$ & $4.4 \pm 0.98^{* * \# \#}$ & $1.7 \pm 0.56^{\# \#}$ \\
H-ATG+ MI/R & $91.8 \pm 8.17^{* * \#}$ & $6.8 \pm 3.33^{\# \#}$ & $4.7 \pm 1.94^{\# \#}$ \\
\hline
\end{tabular}




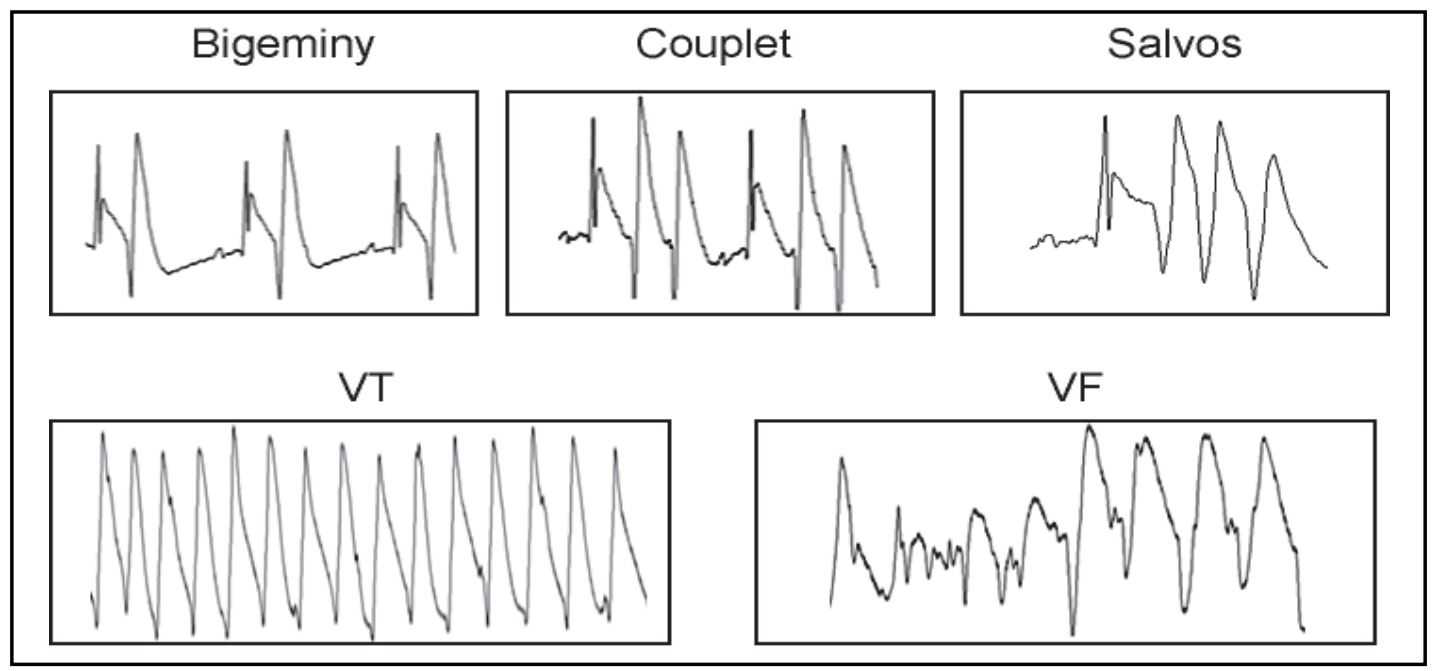

Fig. 1. Representative examples of different ventricular arrhythmias during the 30 min ischemia. VT, ventricular tachycardia; VF, ventricular fibrillation.

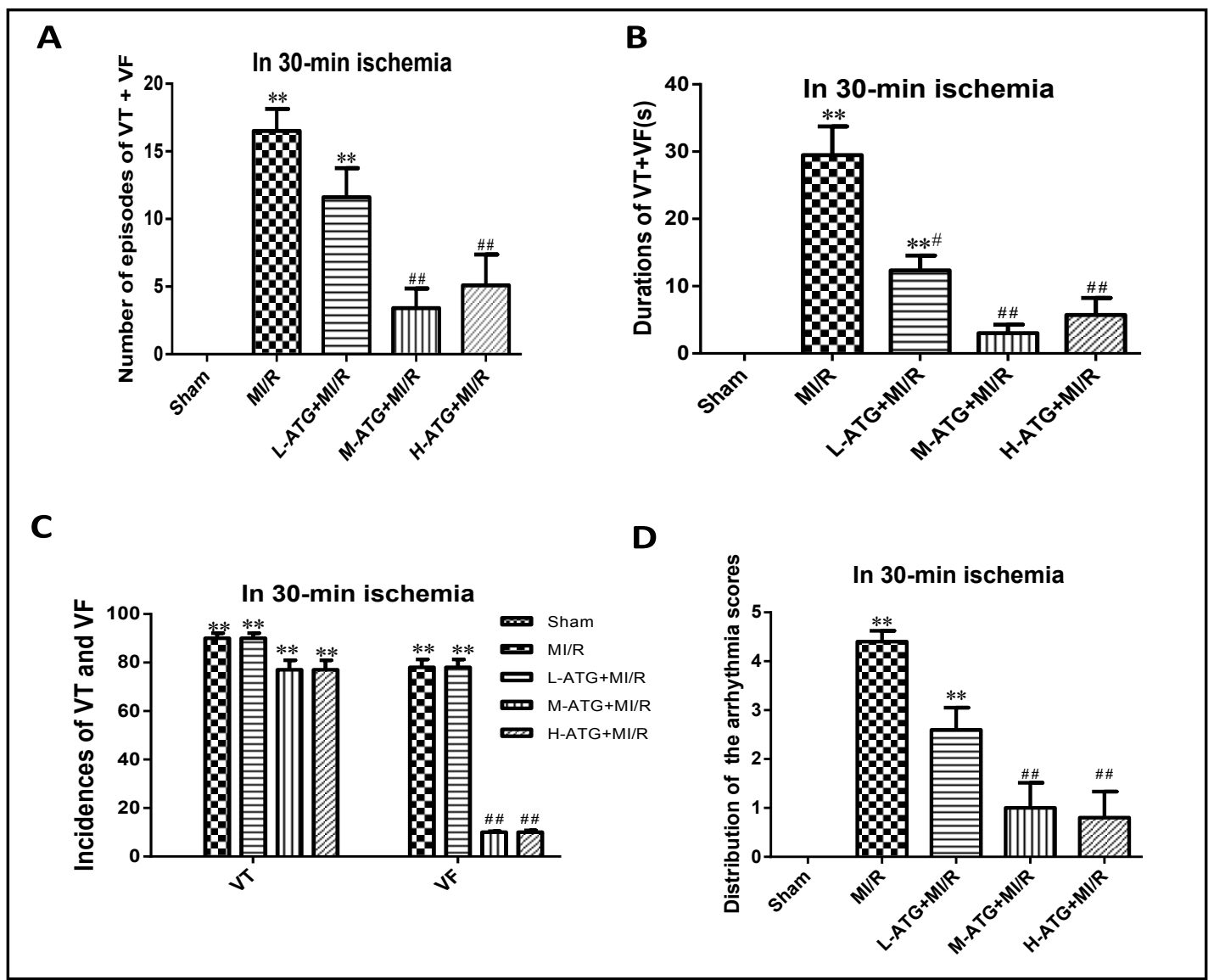

Fig. 2. Parameters of VT + VF during 30-min ischemia. (A) Number of episodes of VT + VF during 30min ischemia. (B) Durations of VT+VF during 30-min ischemia. (C) Incidents of VT and VF during 30-min ischemia. (D) Distribution of the arrhythmia scores during 30-min ischemia. Data were expressed as mean $\pm \mathrm{SE}\left(\mathrm{n}=10\right.$ in each group).VT, ventricular tachycardia; VF, ventricular fibrillation. ATG, arctigenin. ${ }^{* *} \mathrm{P}<0.01$ vs the sham group, ${ }^{\# \#} \mathrm{P}<0.01$ vs MI/R group. 


\section{Cellular Physiology Cell Physiol Biochem 2018;49:728-742 \begin{tabular}{ll|l} 
DOI: 10.1159/000493038 & $\begin{array}{l}\text { O 2018 The Author(s). Published by S. Karger AG, Basel } \\
\text { www.karger.com/cpb }\end{array}$
\end{tabular} \\ Yang et al:: Arctigenin Attenuates Arrhythmias by decreasing Oxidative Stress}

\section{Results}

Ventricular arrhythmias and number of episodes of VEBs/min during 30-min ischemia

In the MI/R model, severe ventricular arrhythmias peaked at 5-25 min after coronary artery ligation. There were different types of ventricular arrhythmias during the 30-min ischemia in the MI/R model (Fig. 1). The numbers of episodes of ventricular ectopic beat (VEB) within 30-min ischemia were significantly different ( $\mathrm{P}>0.05)$, as shown in Table 1.

\section{Numbers and durations of episodes of VT $+V F$ during 30-min ischemia}

The numbers of episodes of VT + VF in the groups pretreated with medium and high doses of ATG $(3.4 \pm 1.5$ and $5.1 \pm 2.3$ ) were significantly fewer than those in the MI/R group $(16.5 \pm 1.6, \mathrm{P}<0.01)$. However, the number of episodes of $\mathrm{VT}+\mathrm{VF}$ in the ATG low dose group $(11.6 \pm 2.2)$ during acute MI was not different from the MI/R group $(16.5 \pm 1.6, \mathrm{P}>0.05)$ (Fig. 2A). The durations of VT + VF during 30 min ischemia in ATG groups pretreated with low, medium and high doses were $12.3 \pm 2.2 \mathrm{~s}, 3.0 \pm 1.2 \mathrm{~s}$ and $5.7 \pm 2.5 \mathrm{~s}$, respectively, which were significantly reduced compared to those in MI/R group (29.5 $\pm 4.3 \mathrm{~s}, \mathrm{P}<0.01)$ (Fig. 2B).

Incidences of VT $+V F$ and severity of arrhythmias during 30-min ischemia

The incidence of VF $(1 / 10,10.0 \% ; 1 / 10,10.0 \%)$ in groups pretreated with medium and high doses of ATG was significantly lower than those in the MI/R group (8/10, 80.0\%, $\mathrm{P}<0.05)$. But there was no significant difference in VT incidence $(8 / 10,80.0 \%$ in medium dose of ATG group ; 8/10, 80.0\% in high dose of ATG group, vs. 9/10, 90\% in MI/R group, P $>0.05)$. In the group receiving a low dose of ATG, the incidence of VF and VT $(7 / 10,70.0 \%$; $9 / 10,90.0 \%$, respectively) during acute MI was no different from those in MI/R group $(8 / 10,80.0 \% ; 9 / 10,90.0 \%$, respectively, $\mathrm{P}>0.05)$ (Fig. 2C). The severity of ventricular arrhythmias was significantly attenuated by ATG administered in medium and high doses of ATG compared to those in the MI/R group ( $<<0.05)$ (Fig. 2D).

Number of episodes of VT $+V F$ during 120-min reperfusion

The numbers of episodes of VT + VF in groups pretreated with medium and high doses of ATG $(0.3 \pm 0.2$ and $0.2 \pm 0.1$, respectively) were significantly lower than those in MI/R group $(2.4 \pm 0.2, \mathrm{P}<0.01)$. However, the numbers of episodes of $\mathrm{VT}+\mathrm{VF}$ in the group receiving a low dose of ATG $(2.2 \pm 0.4)$ during acute MI were not different from those in the MI/R group $(\mathrm{P}>0.05)$ (Fig. 3A).

\section{Durations of VT + VF and severity of arrhythmias during 120-min reperfusion}

The durations of VT + VF in the groups of rats receiving medium and high doses of ATG $(0.4 \pm 0.3 \mathrm{~s}$ and $1.5 \pm 0.8 \mathrm{~s}$, respectively) were significantly shorter than those in MI/R group $(5.1 \pm 0.9 \mathrm{~s}, \mathrm{P}<0.01)$. But the duration of VT $+\mathrm{VF}$ in the ATG low dose group $(4.5 \pm$ $0.8 \mathrm{~s}$ ) during acute MI was not different from those in the MI/R group (P > 0.05) (Fig. 3B). Pretreatment of ATG in a low dose did not produce detectable changes in the severity of ventricular arrhythmias during acute MI compared to those in MI/R group $(\mathrm{P}<0.05)$ (Fig. 3C).

$A T G$ reduced the myocardial infarction area induced by $M I / R$

Myocardial infarction area was increased significantly in the MI/R group and the group receiving ATG in a low dose. However, groups receiving medium and high doses of ATG pretreatments showed a significant reduction in infarction area compared with the MI/R group $(\mathrm{P}<0.01)$ (Fig. 4). 


\section{Cellular Physiology Cell Physiol Biochem 2018;49:728-742 \begin{tabular}{ll|l} 
DOI: 10.1159/000493038 & O 2018 The Author(s). Published by S. Karger AG, Basel \\
wwww.karger.com/cpb
\end{tabular} \\ Yang et al.: Arctigenin Attenuates Arrhythmias by decreasing Oxidative Stress}

ATG elevated antioxidant enzymes SOD, GSH-PX activities and decreased MDA content

To investigate whether or not ATG affects oxidative stress damage we evaluated the activities of antioxidant enzymes SOD and GSH-Px and the content of MDA. The activities of SOD and GSH-Px were significantly lower in the MI/R group compared to the sham group $(P<0.01)$. However, significant elevation of SOD and GSH-Px activities were found in the groups receiving medium and high doses of ATG compared to the MI/R group $(\mathrm{P}<0.01)$. The content of MDA is an index of lipid peroxidation. This was increased significantly after myocardial I/R injury $(\mathrm{P}<0.01)$. ATG pretreatments in medium and high doses significantly decreased the MDA content compared with the MI/R group ( $<0.01$ ) (Fig. 5).

Fig. 3. Parameters of $\mathrm{VT}+\mathrm{VF}$ during 120-min reperfusion. (A) Number of episodes of VT + VF during 120-min reperfusion. (B) Durations of VT+VF during 120min reperfusion. (C) Distribution of the arrhythmia scores during 120-min reperfusion. Data were shown as mean \pm SE ( $n=10$ in each group). VT, ventricular tachycardia; VF, ventricular fibrillation. ATG, arctigenin. ${ }^{* *} \mathrm{P}<0.01$ vs the sham group, ${ }^{\# \#} \mathrm{P}<0.01$ vs the $\mathrm{MI} / \mathrm{R}$ group.

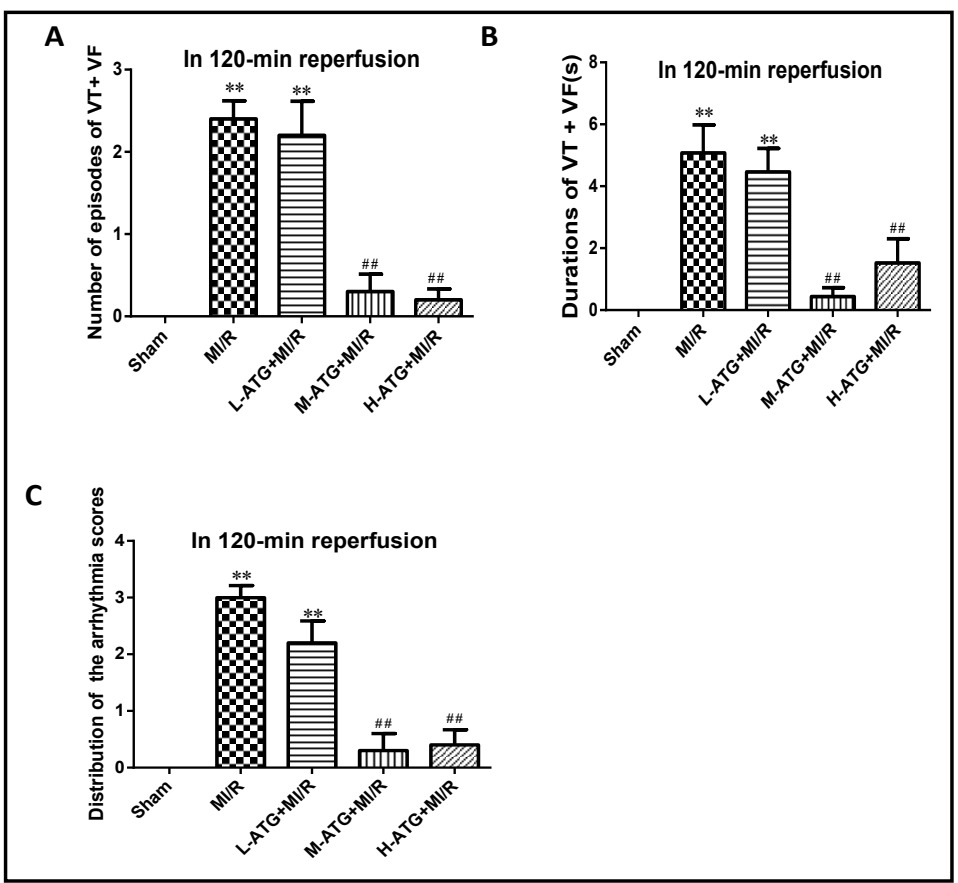

Fig. 4. Effect of ATG on the myocardial infarction area induced by MI/R. (A) Histological sections were stained with Evans blue and TTC. Evans blue stained area (blue staining, non ischemic area), TTC stained area (red staining, ischemic area) and no TTC stained area (white, infarct area) were analyzed with a digital imaging system by computer. (B) Summarized myocardial infarct area in different groups. Data were expressed as mean $\pm \mathrm{SE}(\mathrm{n}=$ 10 in each group). ATG, arctigenin. \#\# $\mathrm{P}<0.01$ vs $\mathrm{MI} / \mathrm{R}$ group.

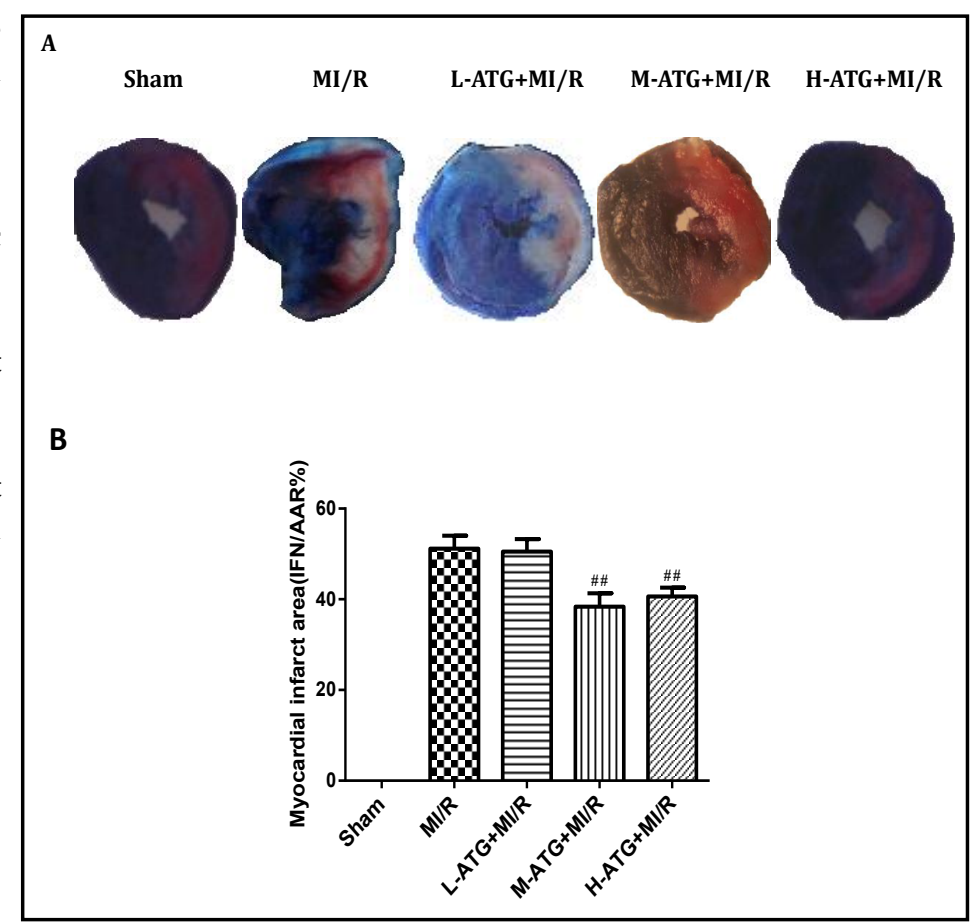




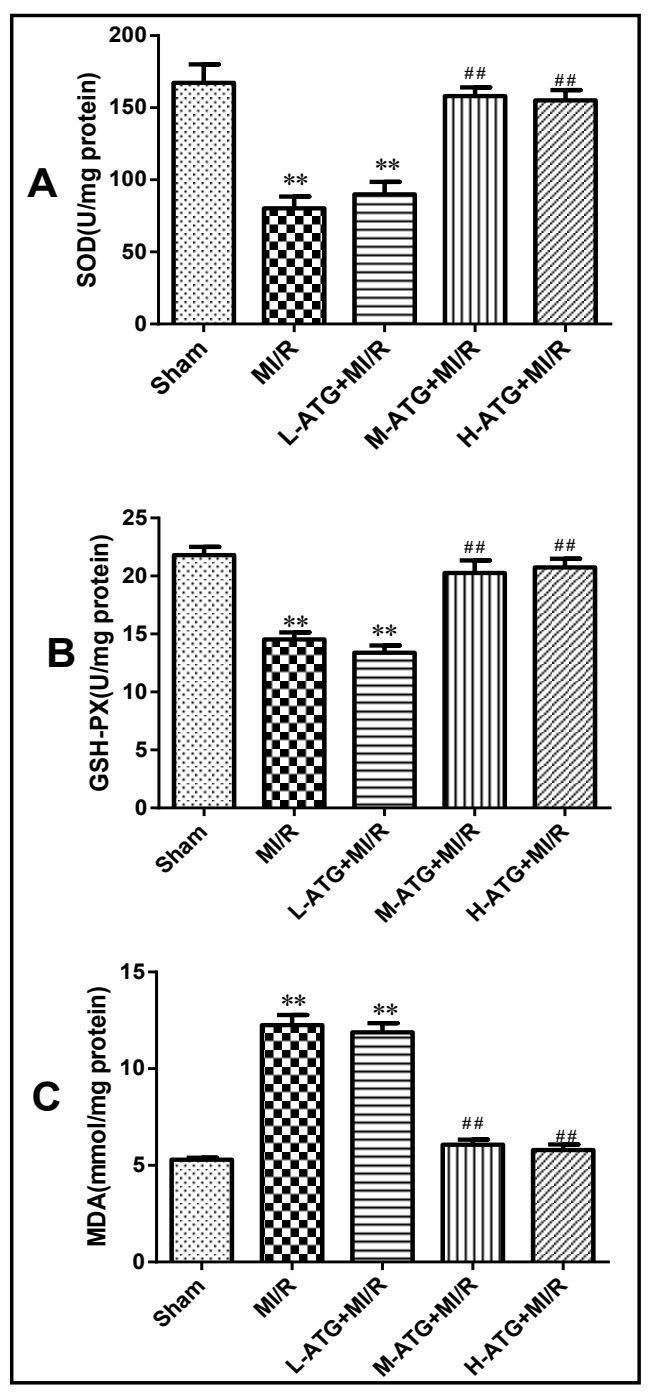

Fig. 5. ATG ameliorates oxidative stress after myocardial ischemia/ reperfusion injury. ATG significantly enhanced (A) superoxide dismutase (SOD) and (B) glutathione peroxidase (GSHPX) activities, and decreased (C) the content of malondialdehyde (MDA) in ischemia/ reperfusion rats hearts. Data were expressed as mean $\pm S E$ ( $n=10$ in each group). ATG, arctigenin. ${ }^{* *} \mathrm{P}<0.01$ vs. the sham group,

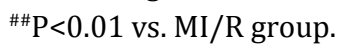

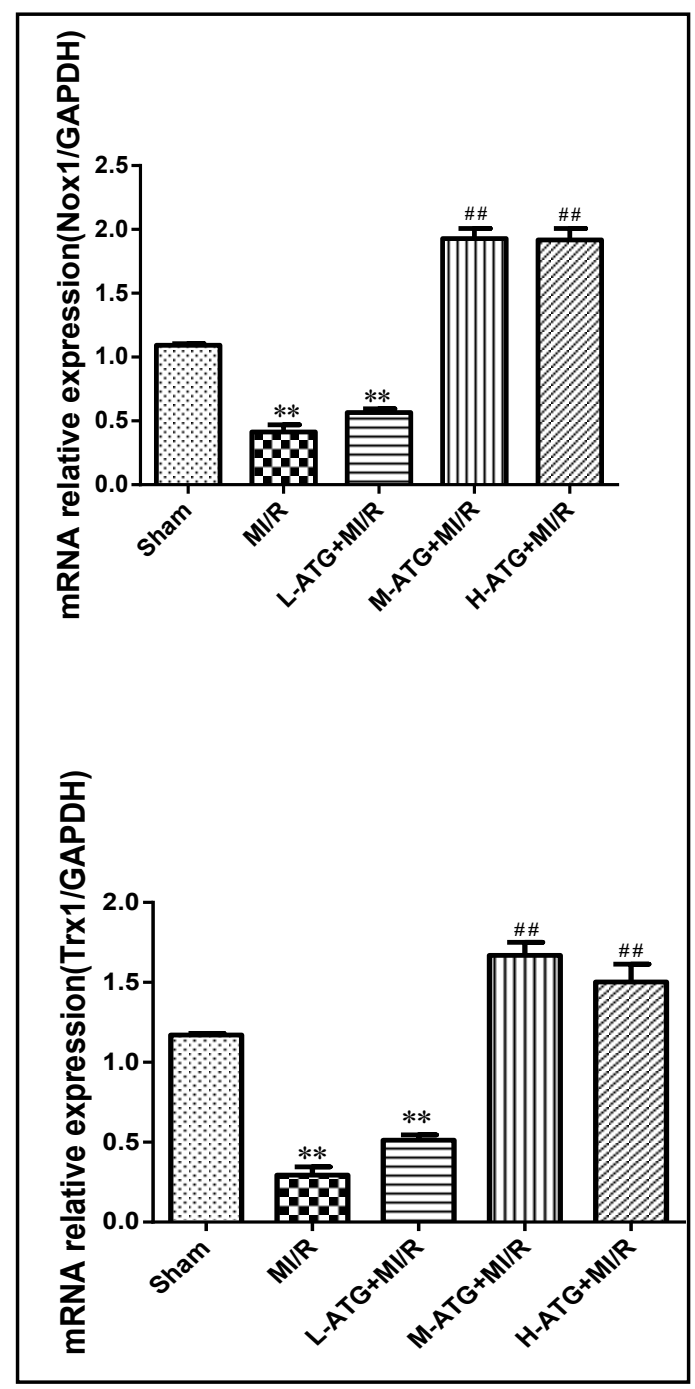

Fig. 6. Effect of ATG on Nox1 and Trx1 mRNA expression in the ischemia area in the heart subjected to MI/R. Data were expressed as mean $\pm \mathrm{SE}(\mathrm{n}=3)$. ATG, arctigenin. ${ }^{* *} \mathrm{P}<0.01$ vs. the sham group, ${ }^{\# \#} \mathrm{P}<0.01$ vs. MI/R group.

ATG up-regulated Nrf2, Trx1 and Nox1 expressions in the ischemia area in the heart subjected to $M I / R$

To investigate which antioxidant stress signaling pathway related to antioxidant enzymes SOD, GSH-Px and MDA, we analyzed the Nox1, Nrf2 and Trx1 expression, which were condidered to be antioxidant stress protein. Real-time PCR showed that the mRNA expression levels of Nox1 and Trx1 were down-regulated in the ischemia area in MI/R group. ATG up-regulated Nox1 and Trx1 mRNA expression in the ischemia area in the heart subjected to MI/R (P < 0.01) (Fig. 6). Then, to further study the effect of ATG on Nox1 and Trx1 mediated signaling, Nrf2, an upstream molecule of Trx1 was also determined by 


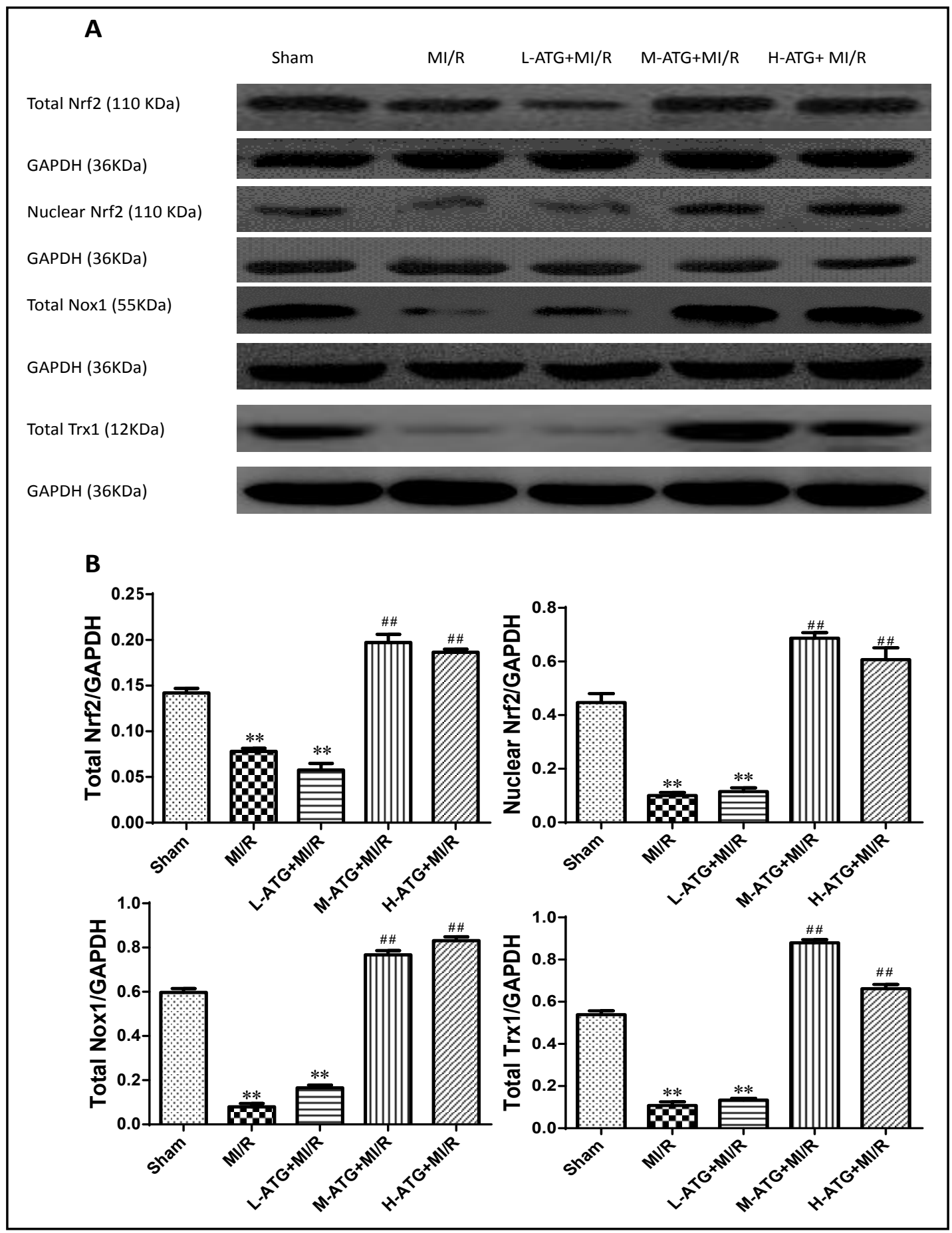

Fig. 7. Effect of ATG on Nrf2, Nox1 and Trx1 expression in the ischemia area in the heart subjected to MI/R. (A)Representative total protein expression of Nrf2, Trx1 and Nox1 in the ischemia area in the heart subjected to MI/R by western blotting.(B) Quantitative densitometry analysis of Nrf2, Trx1 and Nox1 with GAPDH as an internal standard. Data were expressed as mean $\pm \mathrm{SE}(\mathrm{n}=3)$. ATG, arctigenin. ${ }^{* *} \mathrm{P}<0.01$ vs. the sham group, ${ }^{\# \#} \mathrm{P}<0.01$ vs. MI/R group. 


\section{Cellular Physiology Cell Physiol Biochem 2018;49:728-742 \\ \begin{tabular}{l|l|l} 
DOI: 10.1159/000493038 & $\begin{array}{l}\text { O 2018 The Author(s). Published by S. Karger AG, Basel } \\
\text { www.karger.com/cpb }\end{array}$
\end{tabular} \\ Yang et al.: Arctigenin Attenuates Arrhythmias by decreasing Oxidative Stress}

Western blot. The results showed that the expressions of Nrf2, Nox1 and Trx1 were downregulated in the ischemia area in MI/R group. Administration of ATG significantly increased Nrf2, Nox1 and Trx1 expressions ( $<<0.01)$ (Fig. 7).

\section{Discussion}

In this study, we used a classic acute myocardial ischemia/reperfusion damage model to explore the roles of ATG. According to the World Health Organization (WHO) report in 2016, the ischemic heart disease accounted for 2.3 million deaths annually. Systemic thrombolysis and primary percutaneous coronary intervention (PCI) have been used to recover the perfusion, with the latter being the most successful, as it allows reestablishment of the blood flow in the cardiac zones affected by the occlusion of a branch of the coronary artery. As a consequence of this procedure, the ischemic zone is reperfused giving rise to an ischemia/reperfusion event that generates increased production of ROS [18]. This widely performed procedure enhances previously established tissue damage (lethal reperfusion). Effective drugs of ischemia/ reperfusion could be used to measure the effectiveness of novel cardioprotective strategies to prevent lethal reperfusion [4]. Under physiological conditions, ROS is generated at low levels and is widely recognized as important mediators of cell growth, adhesion, differentiation, senescence, and apoptosis $[19,20]$. Under pathologic conditions such as ischemia/reperfusion, their overproduction leads to oxidative stresscausing cell damage which may lead to DNA oxidation, promoting chain reactions of membrane lipid peroxidation and alterations in membrane fluidity [21, 22]. Antioxidants constitute the foremost defense against the toxicity associated with free radicals. It is known that plasma antioxidant capacity decreases and oxidative/antioxidative balance shifts to the oxidative balance in patients with myocardial ischemia.

Reperfusion of ischemic myocardium further aggravates tissue damage induced by ischemia despite providing cells with oxygen and trophic substances. This damage is partly due to the migration of neutrophils from the vasculature to the tissue and the production of ROS $[23,24]$. Superoxide anion is a type of major ROS in ischemia/reperfusion of vascular tissue that originates from the catalysis of NADPH oxidase in the neutrophil [24] or leakage of the electron transport chain in the mitochondria [25]. Since the heart is particularly susceptible to oxidative damage, ischemia/reperfusion-triggered oxidative stress induces cellular apoptosis in the myocardium, which may be attenuated by free radical scavengers [26]. Ischemia/reperfusion injury is one of the most significant etiological factors of heart dysfunction, indicating reperfusion as a key trigger of a number of events leading to myocardial dysfunction associated with ischemia/reperfusion injury [27]. ROS was reportedly produced mostly at reperfusion of the ischemic organ rather than during ischemia [28]. Oxidative stress due to ROS is thought to play a considerable role in ischemia/reperfusion injury that impairs cardiac function. Reactive oxygen species, which can be produced from reperfusion of the ischemic myocardium $[29,30]$, contribute to cardiac ischemia/reperfusion injury. ROS causes the oxidation of DNAs, membranous phospholipidsproteins, and these are implicated in the pathogenesis of ischemia/reperfusion injury, degenerative disease, carcinogenesis and aging. In particular, ROS has the potential to injure cardiac myocytes, endothelial cells and initiate chemical reactions in ischemia/reperfusion injury. Reperfusion of the ischemic heart might result in ROS generation [30] and could be associated with myocardial "stunning" after reversible ischemia/reperfusion injury [31]. Mitochondrial DNA damage is induced by ROS, which leads to the generation of more ROS [32], and perhaps a burst of ROS production. Therefore, the large amount of ROS produced in myocytes after ischemia/reperfusion injury might be mediated in part by myocardial dysfunction (stunning). Reperfusion of the heart after a period of ischemic might lead to potentially lethal arrhythmias. The most important causes of sudden death following spontaneous restoration of integrate flow remain VT and VF [33]. Oxygen-derived free radicals might play a key role in the genesis of ventricular arrhythmias [34]. 


\section{Cellular Physiology Cell Physiol Biochem 2018;49:728-742

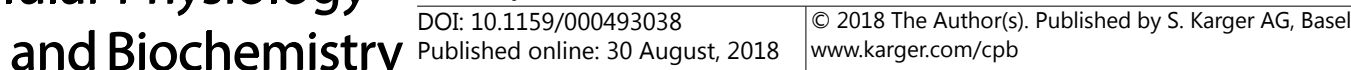 \\ Yang et al.: Arctigenin Attenuates Arrhythmias by decreasing Oxidative Stress}

ATG is an active lignin isolated from Arctium lappa and has anti-inflammation, immunemodulatory, anti-microbial, anti-viral, anti-proliferative, anti-platelet aggregation, anti-carcinogenic and vasodilatory properties [35]. Bae et al. found that ATG could block hydrogen peroxide-induced senescence and cell death though micro RNA expression changes in human dermal papilla cells, which reveal ATG had the anti-oxidative property [35]. Recently, Zhao and colleagues showed that ATG could inhibit aconitine-induced arrhythmia by regulating multi-ion channels, which prompted ATG could be a potential anti-arrhythmic agent [36]. Jeong had reported that ATG can inhibit intracellular ROS production by increasing hemeoxygenase-1 gene expression in rat primary astrocytes [12]. Chang revealed ATG induced endothelial nitric oxide synthase and attenuates subarachnoid hemorrhage-induced vasospasm through PI3K/Akt Pathway in a rat model [13]. ATG even had neuroprotective effect by anti-neuroinflammation and anti-oxidative stress induced by rotenone [15]. However, little is known about the effects and mechanisms of ATG on myocardial ischemia/reperfusion-induced ventricular arrhythmias.

In our research, we first observed the effects of ATG on cardiac function in myocardial ischemia/reperfusion-induced ventricular arrhythmias model. We found that the medium and high doses of ATG can decrease numbers and durations of episodes of VT + VF during 30-min ischemia. Except this, the incidence of VF, the numbers of episodes of VT + VF during 120-min reperfusion, the durations of VT + VF during 120-min reperfusion, the myocardial infarction area induced by ischemia/reperfusion were significantly decreased after treatment with medium and high doses of ATG. The severity of ventricular arrhythmias was significantly attenuated by ATG. Then we focused on the mechanisms of ATG on myocardial ischemia/ reperfusion-induced ventricular arrhythmias. To investigate whether or not ATG affects oxidative stress damage we evaluated the activities of antioxidant enzymes SOD and GSH-Px and the content of MDA. Our results indicated that the elevated level of MDA was markedly decreased by treatment with medium and high doses of ATG, indicating that the cardioprotection conferred by ATG may be attributed to attenuating lipid peroxidation following myocardial ischemia/reperfusion. SOD along with CAT (catalase) and GSH-Px, the preventive antioxidants, play a very important role in protection against lipid peroxidation [37]. Decreased activities of SOD and CAT in the erythrocyte in acute myocardial infarction patients have been reported [38,39], likely due to inactivation by cross-linking or to exhaustion of the enzymes by increased peroxidation [40]. GSH-Px catalyzes peroxide reduction utilizing GSH as the substrate and converting it to GSSG. Its activity has been reported to be decreased in erythrocytes of acute myocardial infarction patients [41]. GSH acts both as a substrate in the scavenging reaction catalyzed by GPx and as a scavenger of vitamins $\mathrm{C}$ and $\mathrm{E}$ radicals. A lowered serum concentration of reduced glutathione (GSH) has been considered the predictor of coronary restenosis after percutaneous coronary intervention, and its deficiency could lead to pronounced postreperfusion syndrome [42]. Decreased GSH concentration may also contribute to decreased GSH-Px activity because GSH is one of its substrates [43]. The overproduction of ROS can be detoxified by endogenous antioxidants, causing their cellular stores to be depleted [44]. SOD and GSH-Px are thought to be two dominant enzymes acting as free radical scavengers that could prevent ROS generation [45]. SOD scavenges the superoxide anion radical (O2) by catalyzing its dismutation to $\mathrm{H} 2 \mathrm{O} 2$, which is scavenged to water by GSH-Px at the expense of glutathione [46]. In this study, ATG appeared to effectively stimulate the activities of SOD and GSH-Px. Our data suggests that ATG protects myocardial ischemia/reperfusion injury through the amelioration of oxidative stress.

Finally, we investigated which antioxidant stress signaling pathway related to antioxidant enzymes SOD, GSH-Px and MDA. Cellular oxygenation is a dynamic process, which is reduced in various physiologic and pathophysiologic conditions. Barančík revealed that Nrf2 as a key player of redox regulation in cardiovascular diseases. The oxidative stress plays an important role in the development of cardiovascular diseases (CVD). In CVD progression, an aberrant redox regulation was observed. In this regulation levels of ROS play an important role in cellular signaling, where Nrf2 is the key regulator of redox homeostasis. Keap1-Nrf2ARE system regulates a great set of detoxificant and antioxidant enzymes in cells after ROS

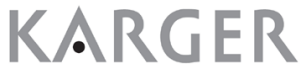




\section{Cellular Physiology Cell Physiol Biochem 2018;49:728-742

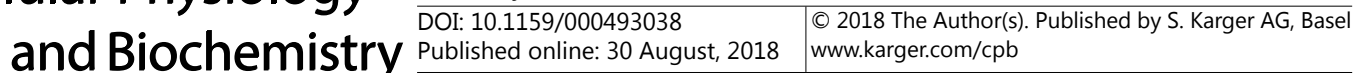 \\ Yang et al.: Arctigenin Attenuates Arrhythmias by decreasing Oxidative Stress}

and electrophiles exposure [47]. Howden demonstrated the relationship between Nrf2 and Ischemia-Reperfusion injury [48]. The myocardium is particularly vulnerable to ischemic injury, because oxygen uptake at any given time during cellular perfusion is around $80 \%$, and therefore cardiac myocytes are unable to significantly increase oxygen uptake from arterial blood when blood flow is severely compromised by vascular constriction (e.g. atherosclerotic plaque thrombosis or vasospasm, leading to ischemia). When blood flow is restored however, a substantial inflammatory response is induced [49], significantly increasing oxidative stress, which can overwhelm antioxidant defenses resulting in cardiac dysfunction from cell damage or death. This process makes Nrf2 an important candidate for resistance to ischemia/reperfusion injury. Conversely, in rat hearts, 30 minutes of left anterior descending coronary artery occlusion resulted in a reduction in Nrf2 nuclear protein, which was prevented by ischemic preconditioning of the myocardium [50]. This finding is very important as it suggests that in order for Nrf2 to initiate antioxidant defenses against reperfusion-induced oxidative stress, the length of the prior ischemic phase may be a critical factor. Early rescue from ischemia may attenuate Nrf2 responses to oxidative stress upon reperfusion, reducing protection from reperfusion-induced oxidative stress. Alternatively, ischemic preconditioning may act as an "early warning" signal and activate Nrf2 prior to a prolonged ischemic event. Acute activation of $\mathrm{Nrf} 2$ has been shown as cardioprotective following ischemia reperfusion. In our study, we analyzed the Nrf2, Trx1 and Nox1 protein expression, which were thought antioxidant stress protein. The results from real-time PCR showed that ATG up-regulated Trx1 and Nox1 expression in the ischemia area in the heart subjected to myocardial ischemia/reperfusion. We further found that the administration of ATG significantly increased Nrf2, Trx1 and Nox1 protein expressions. Nrf2 stability is dependent on cellular redox state. This ROS-dependent transcription factor is induced in response to oxidative stress, which is likely caused by increased levels of endogenously generated ROS [9]. Nrf2 induces transcription of cytoprotective enzymes, such as Trx1 [11, 51-54].

In summary, our research demonstrates that ATG attenuates myocardial ischemia/ reperfusion injury, and that the protective effect of ATG is closely associated with the increase of SOD and GSH-Px activity and decrease of MDA content, which may involve the Nrf2 signaling pathway.

\section{Acknowledgements}

Conceived and designed the experiments: CW, HY. Performed the experiments: JY, YC, YW, MS, ZJ, ZW, RY, JS, Y J. Analyzed the data: JY, CW, H Y. Contributed reagents/materials/analysis tools: JY. Wrote the paper: JY, CW, YL, HY. This work is supported by the National Natural Science Foundation of China (No.81770407, No.31171097, No.31271223, No.31671184, No.2012CB518200 and No.81603676), the Grant of Science and Technology Activities for Excellent Returned Chinese Scholars (No.C2015005004), the Natural Science Foundation of Hebei Province (No.C2018206277, No.H2017206262 and No.C2014206419), the Research Project of Science and Technology of High School of Hebei Province (No.ZD2015005, No.ZD2015007 and No.ZD2016002) and the High-level Talent Support Project of Hebei Province (No.A2017005070).

\section{Disclosure Statement}

The authors have declared that no competing interests exist. 


\section{Cellular Physiology Cell Physiol Biochem 2018;49:728-742 \begin{tabular}{ll|l} 
and Biochemistry Published onlıne: 30 August, 2018 & $\begin{array}{l}\text { (c) } 2018 \text { The Author(s). Published by S. Karger AG, Basel } \\
\text { www.karger.com/cpb }\end{array}$ \\
\hline
\end{tabular}}

Yang et al.: Arctigenin Attenuates Arrhythmias by decreasing Oxidative Stress

\section{References}

1 Quam L, Smith R, Yach D: Rising to the global challenge of the chronic disease epidemic. Lancet 2006;368:1221-1223.

2 Elahi MM, Kong YX, Matata BM: Oxidative stress as a mediator of cardiovascular disease. Oxid Med Cell Longev 2009;2:259-269.

3 Rodrigo R, Libuy M, Feliu F, Hasson D: Oxidative stress-related biomarkers in essential hypertension and ischemia-reperfusion myocardial damage. Dis Markers 2013;35:773-790.

4 Hausenloy DJ, Yellon DM: Myocardial ischemia-reperfusion injury: A neglected therapeutic target. J Clin Invest 2013;123:92-100.

5 Hirose M, Yamaguchi T, Lin C, Kimoto N, Futakuchi M, Kono T, Nishibe S, Shirai T: Effects of arctiin on phipinduced mammary, colon and pancreatic carcinogenesis in female sprague-dawley rats and meiqx-induced hepatocarcinogenesis in male f344 rats. Cancer Lett 2000;155:79-88.

6 Lee S, Shin S, Kim H, Han S, Kim K, Kwon J, Kwak JH, Lee CK, Ha NJ, Yim D: Anti-inflammatory function of arctiin by inhibiting cox-2 expression via nf-kappab pathways. J Inflamm (Lond) 2011;8:16.

7 Hayashi K, Narutaki K, Nagaoka Y, Hayashi T, Uesato S: Therapeutic effect of arctiin and arctigenin in immunocompetent and immunocompromised mice infected with influenza a virus. Biol Pharm Bull 2011;33:1199-1205.

-8 Matsuzaki Y, Koyama M, Hitomi T, Yokota T, Kawanaka M, Nishikawa A, Germain D, Sakai T: Arctiin induces cell growth inhibition through the down-regulation of cyclin d1 expression. Oncol Rep 2008;19:721-727.

$>9$ Osburn WO, Kensler TW: Nrf2 signaling: An adaptive response pathway for protection against environmental toxic insults. Mutat Res 2008;659:31-39.

10 Kim YC, Masutani H, Yamaguchi Y, Itoh K, Yamamoto M, Yodoi J: Hemin-induced activation of the thioredoxin gene by nrf2. A differential regulation of the antioxidant responsive element by a switch of its binding factors. J Biol Chem 2001;276:18399-18406.

-11 Tanito M, Agbaga MP, Anderson RE: Upregulation of thioredoxin system via nrf2-antioxidant responsive element pathway in adaptive-retinal neuroprotection in vivo and in vitro. Free Radic Biol Med 2007;42:1838-1850.

-12 Jeong YH, Park JS, Kim DH, Kim HS: Arctigenin increases hemeoxygenase-1 gene expression by modulating pi3k/akt signaling pathway in rat primary astrocytes. Biomol Ther (Seoul) 2014;22:497-502.

-13 Chang CZ, Wu SC, Chang CM, Lin CL, Kwan AL: Arctigenin, a potent ingredient of arctium lappa l., induces endothelial nitric oxide synthase and attenuates subarachnoid hemorrhage-induced vasospasm through pi3k/akt pathway in a rat model. Biomed Res Int 2015;2015:490209.

14 Song J, Li N, Xia Y, Gao Z, Zou SF, Kong L, Yao YJ, Jiao YN, Yan YH, Li SH, Tao ZY, Lian G, Yang JX, Kang TG: Arctigenin treatment protects against brain damage through an anti-inflammatory and anti-apoptotic mechanism after needle insertion. Front Pharmacol 2016;7:182.

15 Black SC, Rodger IW: Methods for studying experimental myocardial ischemic and reperfusion injury. J Pharmacol Toxicol Methods 1996;35:179-190.

-16 Imani A, Faghihi M, Sadr SS, Keshavarz M, Niaraki SS: Noradrenaline reduces ischemia-induced arrhythmia in anesthetized rats: Involvement of alpha1-adrenoceptors and mitochondrial $\mathrm{k}$ atp channels. J Cardiovasc Electrophysiol 2008;19:309-315.

17 Hu X, Wu B, Wang X, Xu C, He B, Cui B, Lu Z, Jiang H: Minocycline attenuates ischemia-induced ventricular arrhythmias in rats. Eur J Pharmacol 2011;654:274-279.

18 Maxwell SR: Anti-oxidant therapy: Does it have a role in the treatment of human disease? Expert Opin Investig Drugs 1997;6:211-236.

19 Droge W: Free radicals in the physiological control of cell function. Physiol Rev 2002;82:47-95.

20 Loh KP, Huang SH, De Silva R, Tan BK, Zhu YZ: Oxidative stress: Apoptosis in neuronal injury. Curr Alzheimer Res 2006;3:327-337.

-21 Manzanero S, Santro T, Arumugam TV: Neuronal oxidative stress in acute ischemic stroke: Sources and contribution to cell injury. Neurochem Int 2013;62:712-718.

-22 Taylor JM, Crack PJ: Impact of oxidative stress on neuronal survival. Clin Exp Pharmacol Physiol 2004;31:397-406.

-23 Bolli R: Oxygen-derived free radicals and myocardial reperfusion injury: An overview. Cardiovasc Drugs Ther 1991;5 Suppl 2:249-268. 


\section{Cellular Physiology Cell Physiol Biochem 2018;49:728-742 \begin{tabular}{l|l|l|l} 
DOI: 10.1159/000493038 & O 2018 The Author(s). Published by S. Karger AG, Basel \\
wwww.karger.com/cpb
\end{tabular}

24 Marzocca C, Vannacci A, Cuzzocrea S, Salvemini D, Mannaioni PF, Masini E: Effects of the sod mimetic, $\mathrm{m} 40403$, on prostaglandin production in an in vivo model of ischemia and reperfusion in rat heart. Inflamm Res 2003;52 Suppl 1:S23-24.

25 Boveris A: Mitochondrial production of superoxide radical and hydrogen peroxide. Adv Exp Med Biol 1977;78:67-82.

26 Galang N, Sasaki H, Maulik N: Apoptotic cell death during ischemia/reperfusion and its attenuation by antioxidant therapy. Toxicology 2000;148:111-118.

-27 Masini E, Pierpaoli S, Marzocca C, Mannaioni PF, Pietrangeli P, Mateescu MA, Zelli M, Federico R, Mondovi B: Protective effects of a plant histaminase in myocardial ischaemia and reperfusion injury in vivo. Biochem Biophys Res Commun 2003;309:432-439.

28 Cuzzocrea S, Mazzon E, Dugo L, Caputi AP, Aston K, Riley DP, Salvemini D: Protective effects of a new stable, highly active sod mimetic, m40401 in splanchnic artery occlusion and reperfusion. Br J Pharmacol 2001;132:19-29.

-29 Braunwald E, Kloner RA: Myocardial reperfusion: A double-edged sword? J Clin Invest 1985;76:1713-1719.

-30 Zweier JL, Kuppusamy P, Williams R, Rayburn BK, Smith D, Weisfeldt ML, Flaherty JT: Measurement and characterization of postischemic free radical generation in the isolated perfused heart. J Biol Chem 1989;264:18890-18895.

-31 Burton KP, McCord JM, Ghai G: Myocardial alterations due to free-radical generation. Am J Physiol 1984;246:H776-783.

-32 Ide T, Tsutsui H, Hayashidani S, Kang D, Suematsu N, Nakamura K, Utsumi H, Hamasaki N, Takeshita A: Mitochondrial DNA damage and dysfunction associated with oxidative stress in failing hearts after myocardial infarction. Circ Res 2001;88:529-535.

33 Manning AS, Hearse DJ: Reperfusion-induced arrhythmias: Mechanisms and prevention. J Mol Cell Cardiol 1984;16:497-518.

-34 Meerson FZ, Belkina LM, Sazontova TG, Saltykova VA, Arkhipenko Yu V: The role of lipid peroxidation in pathogenesis of arrhythmias and prevention of cardiac fibrillation with antioxidants. Basic Res Cardiol 1987;82:123-137.

-35 Bae S, Lim KM, Cha HJ, An IS, Lee JP, Lee KS, Lee GT, Lee KK, Jung HJ, Ahn KJ, An S: Arctiin blocks hydrogen peroxide-induced senescence and cell death though microrna expression changes in human dermal papilla cells. Biol Res 2014;47:50.

-36 Zhao Z, Yin Y, Wu H, Jiang M, Lou J, Bai G, Luo G: Arctigenin, a potential anti-arrhythmic agent, inhibits aconitine-induced arrhythmia by regulating multi-ion channels. Cell Physiol Biochem 2013;32:1342-1353.

-37 Scott MD, Lubin BH, Zuo L, Kuypers FA: Erythrocyte defense against hydrogen peroxide: Preeminent importance of catalase. J Lab Clin Med 1991;118:7-16.

-38 Anbarasi K, Vani G, Balakrishna K, Devi CS: Effect of bacoside a on brain antioxidant status in cigarette smoke exposed rats. Life Sci 2006;78:1378-1384.

-39 Kotrikadze N, Alibegashvili M, Zibzibadze M, Abashidze N, Chigogidze T, Managadze L, Artsivadze K: Activity and content of antioxidant enzymes in prostate tumors. Exp Oncol 2008;30:244-247.

-40 Salo DC, Pacifici RE, Lin SW, Giulivi C, Davies KJ: Superoxide dismutase undergoes proteolysis and fragmentation following oxidative modification and inactivation. J Biol Chem 1990;265:11919-11927.

41 Bagatini MD, Martins CC, Battisti V, Gasparetto D, da Rosa CS, Spanevello RM, Ahmed M, Schmatz R, Schetinger MR, Morsch VM: Oxidative stress versus antioxidant defenses in patients with acute myocardial infarction. Heart Vessels 2011;26:55-63.

-42 Serdar Z, Aslan K, Dirican M, Sarandol E, Yesilbursa D, Serdar A: Lipid and protein oxidation and antioxidant status in patients with angiographically proven coronary artery disease. Clin Biochem 2006;39:794-803.

43 Scibior D, Skrzycki M, Podsiad M, Czeczot H: Glutathione level and glutathione-dependent enzyme activities in blood serum of patients with gastrointestinal tract tumors. Clin Biochem 2008;41:852-858.

44 Candelario-Jalil E, Mhadu NH, Al-Dalain SM, Martinez G, Leon OS: Time course of oxidative damage in different brain regions following transient cerebral ischemia in gerbils. Neurosci Res 2001;41:233-241.

45 Niizuma K, Yoshioka H, Chen H, Kim GS, Jung JE, Katsu M, Okami N, Chan PH: Mitochondrial and apoptotic neuronal death signaling pathways in cerebral ischemia. Biochim Biophys Acta 2010;1802:92-99.

46 Chan PH: Reactive oxygen radicals in signaling and damage in the ischemic brain. J Cereb Blood Flow Metab 2001;21:2-14. 


\section{Cellular Physiology Cell Physiol Biochem 2018;49:728-742 \begin{tabular}{ll|l} 
DOI: 10.1159/000493038 & $\begin{array}{l}\text { O 2018 The Author(s). Published by S. Karger AG, Basel } \\
\text { www.karger.com/cpb }\end{array}$ \\
\hline
\end{tabular}}

Yang et al.: Arctigenin Attenuates Arrhythmias by decreasing Oxidative Stress

47 Barancik M, Gresova L, Bartekova M, Dovinova I: Nrf2 as a key player of redox regulation in cardiovascular diseases. Physiol Res 2016;65 Suppl 1:S1-S10.

48 Howden R: Nrf2 and cardiovascular defense. Oxid Med Cell Longev 2013;2013:104308.

49 de Groot H, Rauen U: Ischemia-reperfusion injury: Processes in pathogenetic networks: A review. Transplant Proc 2007;39:481-484.

50 Gurusamy N, Malik G, Gorbunov NV, Das DK: Redox activation of ref-1 potentiates cell survival following myocardial ischemia reperfusion injury. Free Radic Biol Med 2007;43:397-407.

-51 Itoh K, Wakabayashi N, Katoh Y, Ishii T, Igarashi K, Engel JD, Yamamoto M: Keap1 represses nuclear activation of antioxidant responsive elements by nrf2 through binding to the amino-terminal neh2 domain. Genes Dev 1999;13:76-86.

52 Kobayashi M, Yamamoto M: Molecular mechanisms activating the nrf2-keap1 pathway of antioxidant gene regulation. Antioxid Redox Signal 2005;7:385-394.

53 Kensler TW, Wakabayashi N, Biswal S: Cell survival responses to environmental stresses via the keap1nrf2-are pathway. Annu Rev Pharmacol Toxicol 2007;47:89-116.

54 Rushmore TH, Morton MR, Pickett CB: The antioxidant responsive element. Activation by oxidative stress and identification of the DNA consensus sequence required for functional activity. J Biol Chem 1991;266:11632-11639. 